Please do not remove this page

RMIT

UNIVERSITY

\title{
Discrete self-trapping vs. defocusing in nonlinear waveguide arrays
}

Rosberg, Christian; Matuszewski, Michal; Neshev, Dragomir; Sukhorukov, Andrey; Mitchell, Arnan;

Trippenbach, Marek; Austin, Michael

https://researchrepository.rmit.edu.au/esploro/outputs/9921860051801341/filesAndLinks?institution=61RMIT_INST\&index=null

Rosberg, C., Matuszewski, M., Neshev, D., Sukhorukov, A., Mitchell, A., Trippenbach, M., Austin, M., Krolikowski, W., \& Kivshar, Y. (2006). Discrete self-trapping vs. defocusing in nonlinear waveguide arrays. Proceedings of the Conference on Lasers and Electro-Optics/Quantum Electronics and Laser Science Conference (CLEO/QELS 2006), 1-2. https://doi.org/10.1109/CLEO.2006.4628901

Published Version: https://doi.org/10.1109/CLEO.2006.4628901

Repository homepage: https://researchrepository.rmit.edu.au (C) 2006 IEEE

Downloaded On 2023/04/26 17:24:11 +1000 


\title{
Discrete Self-Trapping vs. Defocusing in Nonlinear Waveguide Arrays
}

\author{
Christian R. Rosberg ${ }^{1}$, Michal Matuszewski ${ }^{1,2}$, Dragomir N. Neshev ${ }^{1}$, Andrey A. Sukhorukov ${ }^{1}$, Arnan \\ Mitchell $^{4}$, Marek Trippenbach ${ }^{2,3}$, Michael W. Austin ${ }^{4}$, Wieslaw Krolikowski ${ }^{1}$, and Yuri S. Kivshar ${ }^{1}$ \\ ${ }^{1}$ Nonlinear Physics Centre and Laser Physics Centre, \\ Centre for Ultrahigh-bandwidth Devices for Optical Systems (CUDOS), \\ Research School of Physical Sciences and Engineering, \\ The Australian National University, Canberra ACT 0200, Australia \\ ${ }^{2}$ Institute for Theoretical Physics, Warsaw University, Poland \\ ${ }^{3}$ Soltan Institute for Nuclear Studies, Warsaw, Poland \\ ${ }^{4}$ School of Electrical and Computer Systems Engineering, RMIT University, Melbourne, Australia
}

We predict a sharp crossover from self-defocusing to discrete localization in photonic lattices with defocusing nonlinearity for increasing index contrast. We demonstrate experimentally discrete self-trapping of light by single site excitation in $\mathrm{LiNbO}_{3}$ waveguide arrays.

(C) 2005 Optical Society of America

OCIS codes: $190.4420,190.5940$

Nonlinear periodic photonic structures offer unique possibilities to engineer and control optical wave diffraction and nonlinear self-action of light ${ }^{1}$. In this paper, we study the self-action of a narrow Gaussian beam propagating in a nonlinear defocusing medium with a periodic refractive index modulation in the transverse direction. We demonstrate that a sharp transition from self-defocusing to discrete self-trapping occurs when the depth of the index modulation is increased, and we observe experimentally the formation of a self-trapped state from single-site excitation in a nonlinear defocusing waveguide array.
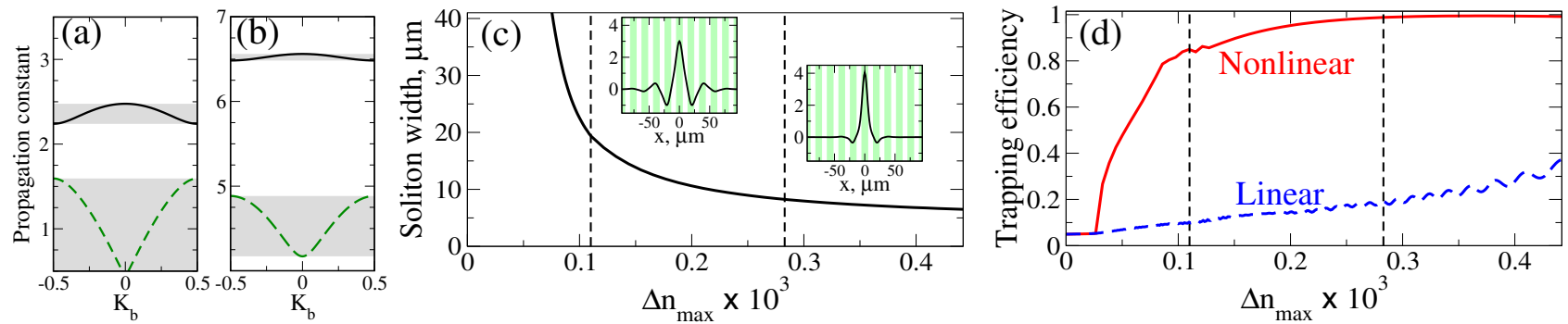

Fig. 1. (a,b) Bandgap structure for a refractive index contrast of $1.1 \times 10^{-4}$ and $2.8 \times 10^{-4}$, respectively. (c) Minimum gap soliton width vs. refractive index contrast. Insets show the soliton profiles corresponding to (a,b). (d) Beam self-trapping efficiency vs. refractive index contrast, calculated as the power fraction remaining in the 20 central waveguides at the output for optimized input power (solid) compared with low power linear diffraction (dashed).

Nonlinear propagation of light in periodic photonic structures is commonly described by a discretizing tight-binding approximation, $i d a_{n} / d z+\beta a_{n}+C\left(a_{n-1}+a_{n+1}\right)+\gamma\left|a_{n}\right|^{2} a_{n}=0$, where $z$ is the propagation coordinate, $a_{n}(z)$ is the $n$-th mode amplitude, $\beta$ is the propagation constant, $C$ is the coupling coefficient, and the last term accounts for nonlinear mode detuning. In the framework of this discrete model, there is a full equivalence between the two types of nonlinearities since the above equation remains unchanged after the transformation: $a_{n} \rightarrow(-1)^{n} a_{n}^{*}$ and $\gamma \rightarrow-\gamma$. Provided only a single waveguide is excited, the only difference is in the phase structure of the nonlinear localized solution. Indeed, a self-focusing nonlinearity $(\gamma>0)$ supports light self-localization in the form of unstaggered discrete solitons $s^{1,2}$, whereas a defocusing nonlinearity $(\gamma<0)$ can lead to self-trapping in the form of staggered solitons when the amplitude of the modes in neighboring waveguides is out of phase ${ }^{3}$.

The discrete model is generally valid when the contrast of the refractive index modulation is sufficiently large. However, in the case of defocusing nonlinearity, the beam will experience nonlinearly enhanced defocusing instead of self-trapping if the lattice modulation is absent or very weak. In order to study beam propagation beyond the limits of the discrete model we numerically solve a normalized continuous nonlinear Schrödinger equation for the slowly varying field envelope $E(x, z), i \partial E / \partial z+D \partial^{2} E / \partial x^{2}+\mathcal{F}\left(|E|^{2}\right) E+\rho \Delta n(x) E=0$, where $D=z_{s} \lambda /\left(4 \pi n_{0} x_{s}^{2}\right)$ is the diffraction coefficient, $\rho=2 \pi z_{s} / \lambda, x$ is normalized to $x_{s}$, and $z$ is normalized to $z_{s}$. To match our experiments, the linear refractive index change $\Delta n$ is taken as $\Delta n(x)=\xi \sum_{n} \exp \left[-(x-n d)^{2} / w^{2}\right]$, where the waveguide width $w=12 \mu \mathrm{m}$, the waveguide spacing $d=19 \mu \mathrm{m}$, and $\xi$ defines the modulation depth. The refractive index contrast

Citation: Rosberg, C, Matuszewski, M, Neshev, D, Sukhorukov, A, Mitchell, A, Trippenbach, M, Austin, M, Krolikowski, W and Kivshar, Y 2006, 'Discrete self-trapping vs. defocusing in nonlinear waveguide arrays', in Proceedings of the Conference on Lasers and Electro-Optics/Quantum Electronics and Laser Science Conference (CLEO/QELS 2006), California, USA, 21-26 May 2006. 


\section{QFF6.pdf}

is therefore $\Delta n_{\max }=0.442 \xi$ due to overlap of the individual index profiles. Other parameters are: $\lambda=0.532 \mu \mathrm{m}$, $n_{0}=2.234, x_{s}=1 \mu \mathrm{m}, z_{s}=1 \mathrm{~mm}$, and $\mathcal{F}(I)=1.5(1+I)^{-1}$ for photovoltaic defocusing nonlinearity.

The periodic modulation of the refractive index results in the formation of a bandgap structure [Fig. 1(a,b)]. In the case of defocusing nonlinearity, staggered solitons can form at the bottom edge of the first band. The size of the gap [see Fig. 1(a,b)] limits the minimum width of self-trapped beams as shown in Fig. 1(c). For a small index contrast, the narrowest soliton spans several waveguides, but localizes at a single waveguide when the index contrast is increased [see insets in Fig. 1(c)]. We study this transition by modeling the propagation of a narrow Gaussian input beam matching the width of a single waveguide. In Fig. 1(d) we plot, as a function of the index contrast, the relative power that remains in the central section of the array after propagation over a distance of many diffraction lengths (1m). We compare linear diffraction (dashed line) to the effect of nonlinearity (solid line) when the power is optimized to maximize the fraction which remains in the central section of the array. The nonlinear self-action results in beam self-defocusing in the region where the two curves coincide in Fig. 1(d), but a bifurcation appears for increasing index contrast, corresponding to the formation of initially broad gap solitons. As the minimum width of the soliton decreases and approaches that of a single waveguide, self-trapping with almost $100 \%$ efficiency becomes possible, representing a sharp crossover from self-defocusing to discrete self-trapping at a certain index contrast threshold.

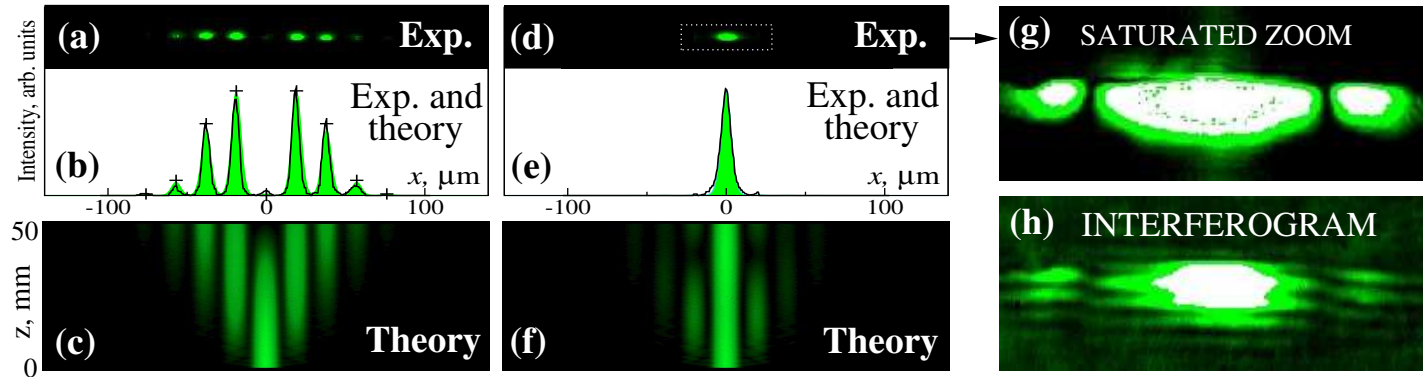

Fig. 2. (a) Output intensity distribution for linear propagation at low laser power $(0.1 \mu \mathrm{W})$. (b) Corresponding intensity profiles: Solid line - experiment; shading - numerical solution of continuous model; crosses - discrete model lattice site amplitudes. (c) Simulated beam intensity along the sample for low input power. (d-f) Same as (a-c) for nonlinear propagation at high laser power $(0.1 \mathrm{~mW})$. (g) Saturated image of the localized state in (d). (h) Interferogram confirming the staggered phase structure of the output beam.

In previous experimental studies ${ }^{4,5}$ of self-localization in periodic structures with defocusing nonlinearity, the input beam was specially prepared to reflect the phase structure of the staggered soliton. However, observation of soliton localization at a single waveguide was not reported. In our experiments, we study single-site discrete self-trapping in an array of closely spaced single mode waveguides fabricated by Titanium indiffusion into a mono-crystal lithium niobate wafer, producing an index contrast $\Delta n_{\max }=2.8 \times 10^{-4}$. The $\mathrm{LiNbO}_{3}$ sample exhibits a strong photovoltaic effect which leads to a self-defocusing nonlinear response. An extraordinarily polarized beam from a cw $\mathrm{Nd} \mathrm{YVO}_{4}$ laser is tightly focused onto a single waveguide at the input of the array. At low laser power $(\sim 0.1 \mu \mathrm{W})$ the probe beam experiences typical discrete diffraction [Fig. 2(a)]. The corresponding output intensity profile in Fig. 2(b, solid line) matches numerical calculations performed using both the discrete model (crosses) and the full continuous model (shading). The simulated propagation inside the array is depicted in Fig. 2(c). When the laser power is increased $(\sim 0.1 \mathrm{~mW})$ the defocusing photovoltaic nonlinearity leads to strong beam localization at a single waveguide [Fig. 2(df)], in a similar way as in focusing waveguide arrays. To confirm that the nonlinear state is indeed localized inside the Bragg reflection gap, and not in the total internal reflection gap, its staggered phase structure is verified by saturated image detection of zero intensity lines [Fig. 2(g)] and by interferometric measurement with a reference wave [Fig. 2(h)].

In conclusion, we have studied the crossover between beam self-defocusing and discrete self-trapping in waveguide arrays with defocusing nonlinearity. We have shown that an abrupt transition is observed in the beam dynamics with the increase of the refractive index, and demonstrated experimentally the generation of strongly localized staggered states in nonlinear defocusing waveguide arrays by single waveguide excitation.

\section{References}

1. D. N. Christodoulides, F. Lederer, and Y. Silberberg, Nature 424, 817 (2003).

2. D. N. Christodoulides and R. I. Joseph, Opt. Lett. 13, 794 (1988).

3. Yu. S. Kivshar, Opt. Lett. 18, 1147 (1993).

4. F. Chen, M. Stepic, C. E. Ruter, D. Runde, D. Kip, V. Shandarov, O. Manela, and M. Segev, Opt. Express 13, $4314(2005)$.

5. J. W. Fleischer, M. Segev, N. K. Efremidis, and D. N. Christodoulides, Nature 422, 147 (2003). 